\title{
EFEITOS DO TIOTIXENE (P-4657 B) EM CRIANÇAS E ADOLESCENTES PSICÓTICOS
}

\author{
EDY S. NAVARRo * \\ ENEIDA BAPTISTETE * \\ MiriaM V. SALDANHA ***
}

\author{
Carlos A. Fonseca ** \\ Julcir M. R. PENNA * \\ Cacilda C. dos Santos*
}

Em trabalho anterior ${ }^{6}$ estudamos o efeito do tiotixene em 11 crianças e adolescentes esquizofrênicos e consideramos os resultados obtidos como satisfatórios para justificar o emprêgo da droga. Resolvemos, por isso, prosseguir na experimentação dêste nôvo neuroléptico com doses mais elevadas (15 a $70 \mathrm{mg} /$ dia) e com maior número de pacientes em sua maioria com psicose esquizofrênica. Os resultados obtidos vieram confirmar aquêles anteriormente registrados ${ }^{1-6}$.

\section{MATERIAL E MÉTODOS}

Aos casos já registrados em trabalho anterior (11 pacientes esquizofrênicos e de idade variável de 9 a 16 anos, com ou sem tratamento prévio) acrescentamos 23 pacientes de ambos os sexos, de idade variável entre 7 a 16 anos, dos quais apenas 6 já haviam feito outro tratamento. No quadro 1 figuram dados relativos à identificação, idade dos doentes, tempo de doença, diagnóstico, tratamentos anteriores, duração do tratamento pelo tiotixene, doses empregadas e resultados nos 34 casos estudados.

Nos pacientes já tratados por outros medicamentos, houve intervalo minimo de 15 dias entre o último tratamento e o inicio da administracão do tiotixene.

Provas laboratoriais (hemograma, provas de função hepática, exame de urina e eletrencefalograma) foram feitas antes, durante a administração da dose máxima e depois do tratamento; foi feito contrôle diário de pulso, pressão arterial e temperatura; o peso foi controlado semanalmente.

A dose inicial de tiotixene foi $3 \mathrm{mg}$ diários, sendo aumentada de $3 \mathrm{mg}$ cada três dias até atingir $15 \mathrm{mg}$ por dia. A dose máxima variou de acôrdo com a resposta clínica obtida (15 a $70 \mathrm{mg}$ por dia). Não foram usadas outras drogas antipsicóticas durante o tempo em que durou êste estudo. Nos casos em que os sintomas extrapiramidais foram mais evidentes foi administrada pequena dose de droga antiparkinsoniana.

O tempo de tratamento variou entre 8 a 20 semanas, de acôrdo com a resposta de cada caso. Durante o tratamento os pacientes foram examinados diàriamente para avaliaçāo dos efeitos clínicos da droga.

Consideramos os resultados de acôrdo com as seguintes gradaçōes: ótimo, quando ocorreu total remissão da sintomatologia psiquica; bom, quando houve

Da Clinica Psiquiátrica da Faculdade de Medicina da Universidade de São Paulo: * Médicos assistentes; ** Médico contratado; *** Médico estagiário. 


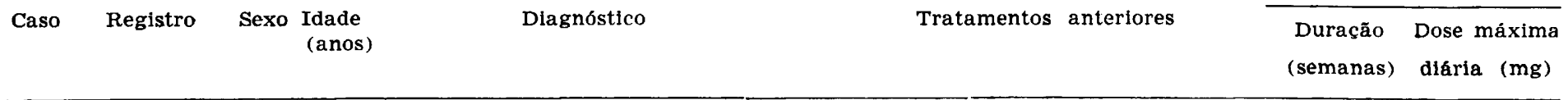

\begin{tabular}{|c|c|c|c|c|c|c|c|}
\hline 1 & 37939 & $\mathbf{F}$ & 14 & Esquizofrenia hebefrenocatatônica. & & 16 & 25 \\
\hline 2 & 36093 & $\mathrm{~F}$ & 14 & Esquizofrenia simples & $\begin{array}{l}\text { Enantato de flufenazina, clorpromazina, } \\
\text { trifluperidol. }\end{array}$ & 16 & 30 \\
\hline $\mathbf{3}$ & $\mathbf{3 7 6 1 0}$ & $\mathbf{F}$ & 16 & Esquizofrenia simples & & 14 & 40 \\
\hline 4 & 33492 & $\mathbf{F}$ & 16 & Esquizofrenia hebefrenocatatônica. & $\begin{array}{l}\text { Eletrochoque, enantato de flufenazina, } \\
\text { clorpromazina, haloperidol. }\end{array}$ & 10 & 35 \\
\hline 5 & 35948 & $\mathbf{F}$ & 16 & Esquizofrenia simples & $\begin{array}{l}\text { Eletrochoque, clorpromazina, haloperi- } \\
\text { dol. }\end{array}$ & 16 & 40 \\
\hline 6 & 30959 & $\mathbf{F}$ & 13 & Esquizofrenia simples & $\begin{array}{l}\text { Insulinoterapia, eletrochoque, clorpro- } \\
\text { mazina, trifluperiodol. }\end{array}$ & 16 & 30 \\
\hline 7 & 30607 & $\mathbf{F}$ & 9 & Esquizofrenia infantil & $\begin{array}{l}\text { Insulinoterapia, enantato de fenazina, } \\
\text { clorpromazina, trifluperidol, levomepro- } \\
\text { mazina, haloperidol. }\end{array}$ & 16 & 20 \\
\hline 8 & 38365 & $\mathbf{F}$ & 15 & Esquizofrenia simples & & 16 & 40 \\
\hline 9 & 38421 & $\mathbf{M}$ & 15 & Esquizofrenia hebefrênica. & & 20 & 40 \\
\hline 10 & 38373 & $\mathbf{M}$ & 15 & Esquizofrenia hebefrênica. & & 20 & 20 \\
\hline 11 & 37099 & M & 15 & Esquizofrenia hebefrênica. & Insulinoterapia, convulsoterapia. & 20 & 40 \\
\hline 12 & 39047 & $\mathbf{F}$ & 16 & Esquizofrenia simples & & 12 & 50 \\
\hline 13 & 39171 & $\mathbf{F}$ & 11 & Psicose epilética & Haloperidol & 8 & 15 \\
\hline 14 & 23094 & M & 8 & Psicose por lesão cerebral. & $\begin{array}{l}\text { Clorpromazina, tioproperazina, enantato } \\
\text { de flufenazina, propericiazina. }\end{array}$ & 10 & 40 \\
\hline 15 & 29417 & $\mathbf{M}$ & 15 & Psicose por lesão cerebral & $\begin{array}{l}\text { Propericiazina, levomepromazina. enan- } \\
\text { tato de flufenazina, tioproperazina. }\end{array}$ & 16 & 70 \\
\hline
\end{tabular}




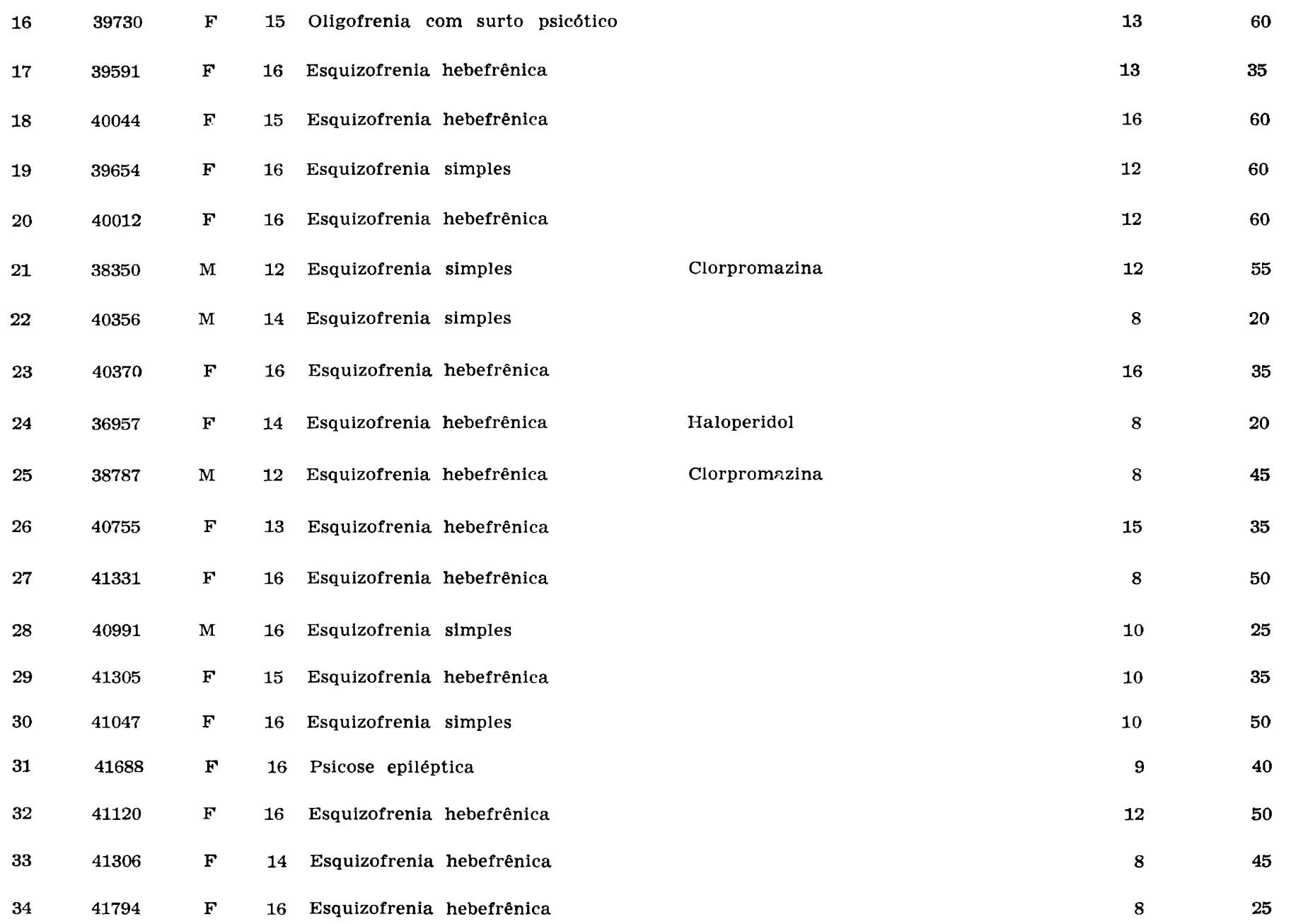

QUADRO 1 - Identificação e idade dos doentes, diagnóstico, tratamentos anteriores, duração do tratamento pelo Tiotixene, e doses empregadas em 34 casos. 
melhora da sintomatologia psiquica que, entretanto, não podia ser observada em todos os sintomas que o paciente apresentava (remissão social); discreto. quando a meihora atingia poucos sintomas (remissão parcial); inalterado, quando nāo havia qualquer melhora da sintomatologia; piorado, quando o quadro psiquico se agravou apesar do tratamento*.

\section{R E S L T A D O S}

Os resultados quantitativos constam dos quadros 2 e 3 .

\begin{tabular}{lcccccc}
\hline & \multicolumn{5}{c}{ Pacientes } & \multicolumn{3}{c}{ Resultados } & Totais \\
\cline { 2 - 6 } & ótimo & bom & disc. & inalt. & piorado \\
\hline \hline c/ trat. prévio & 4 & 1 & 2 & 4 & 1 & 12 \\
s/ trat. prévio & 10 & 5 & 4 & 3 & -12 \\
Totais & 14 & 6 & 6 & 7 & 1 & 34 \\
\hline
\end{tabular}

QUADRO 2 - Resultados globais com tiotixene em 34 casos estudados: disc. = discreto; inalt. = inalterado.

Diagnóstico

Resultados

Totais

ótimo bom disc. inalt. piorado

\begin{tabular}{|c|c|c|c|c|c|c|}
\hline Esquizofrenia hebefrênica & 7 & 4 & 4 & 1 & 1 & 17 \\
\hline Esquizofrenia simples & 4 & 2 & 一 & 5 & - & 11 \\
\hline Esquizofrenia infantil * & - & - & 1 & - & - & 1 \\
\hline Psicose epiléptica & 2 & - & - & - & - & 2 \\
\hline Surto psicótico em oligofrênico & 1 & - & - & - & - & 1 \\
\hline Psicose por lesão cerebral & 一 & - & 1 & 1 & - & 2 \\
\hline Totais & 14 & 6 & 6 & 7 & 1 & $\mathbf{3 4}$ \\
\hline
\end{tabular}

QUADRO 3 - Resultados quanto ao diagnóstico nos 34 casos estudados. * Consideramos esquizofrenia infantil quando a doença se manifestou antes dos 10 anos de idade configurando um quadro clinico próprio que justifica sua separação das formas clássicas.

Os resultados qualitativos constam dos quadros 4,5 e 6 .

* Nota dos autores - Agradecemos à Pfizer Quimica Ltda. o fornecimento do tiotixene (Navane R) utilizado neste ensáio terapêutico. 
Tempo de doença

Resultados

Totais

ótimo bom disc. inalt. piorade

\begin{tabular}{|c|c|c|c|c|c|c|}
\hline Mais de 2 anos & - & 1 & 4 & 2 & 1 & 8 \\
\hline Mais de 1 ano & 4 & -- & 一 & 2 & $\longrightarrow$ & 6 \\
\hline Mais de 6 meses & - & - & - & 3 & 一 & 3 \\
\hline Menos de 6 meses & 10 & 5 & 2 & 一 & - & 17 \\
\hline
\end{tabular}

Quadro 4 - Análìse das relações entre tempo de doença e os resultados.

N.9 casos N.9 remissōes

\begin{tabular}{lll}
\hline \hline Agitação & 18 & 13 \\
Idéias delírantes & 18 & 15 \\
Alucinações & 14 & 12 \\
Negativismo & 18 & 11 \\
Dissociação do pensamento & 16 & 10 \\
Autismo & 29 & 16 \\
Embotamento afetivo & 32 & 15 \\
Apragmatismo & 30 & 16
\end{tabular}

Quadro 5 - Análise dos resultados quanto à sintomatologia psíquica.

Os efeitos colaterais que foram analisados no quadro 6 não estiveram relacionados com determinada posologia da droga, mas tão sòmente com a sensibilidade individual. Não houve alteração significativa no pulso, pressão arterial e temperatura. Nas doses empregadas a tolerância à medicação foi sempre ótima.

\begin{tabular}{lccc}
\hline & intensos & moderados & discretos \\
\hline \hline Extra-piramidais (sindrome parkisoniana) & 3 & 3 & 13 \\
Sonolência & 1 & 2 & 15 \\
Aumento de peso & 3 & 3 \\
Exacerbação do erotismo & 1 & 3 \\
\hline Quadro $6-$ Análise dos resultados quanto aos efeitos colaterais.
\end{tabular}


Análise dos resultados quanto aos exames de laboratorio - o hemograma, as provas de função hepática e o exame de urina não revelaram antes, durante e depois do tratamento qualquer alteração significativa.

Não houve alteração dos exames eletrencefalográficos em 30 pacientes. Em um caso em que havia paroxismos de ondas lentas atípicas (espicula-onda lenta atipica) antes do tratamento, os traçados durante e depois do uso da droga foram normais, tendo a paciente permanecido inalterado em sua sintomatologia (caso 5). Em outro (caso 6) com eletrencefalograma normal antes do tratamento, o tracado passou a revelar anormalidades (foco temporal direito, ondas "sharp") durante e depois do uso do tiotixene; é importante assinalar que esta paciente já havia tido crises convulsivas na infância e que voltou a apresentá-las durante e depois do uso do tiotixene, fato que coincidiu com a melhora do quadro psicótico. Em um terceiro paciente (caso 13) o eletrencefalograma era anormal anteriormente (onda "sharp" na região temporal esquerda); durante o tratamento o traçado normalizou-se e, após o tratamento, o traçado passou a revelar ondas "sharp" em ambas as regiões temporais, coincidindo com melhora da sintomatologia do paciente. Finalmente em um quarto caso (caso 19) o eletrencefalograma anterior ao tratamento era normal; durante e depois do uso da droga, revelou disritmia paroxistica bilateral síncrona; êste paciente não obteve benefício com o tratamento.

\section{RESUMO E CONCLUSOES}

Foram estudados os efeitos do tiotixene (P-4657B) em 34 crianças e adolescentes psicóticos dos quais 29 eram esquizofrênicos, tendo 17 dêles menos de um ano de tempo de doença e 12 mais de um ano de doença. Foram obtidos bons resultados em 20 casos com nítida melhora na sintomatologia psiquica. Os efeitos colaterais foram de pequena intensidade. Os exames subsidiários (hemograma, provas de função hepática e eletrencefalograma) não revelaram alterações significativas.

Assim o tietixene confirmou sua atividade antipsicótica não só nos casos em que foi o primeiro tratamento instituído como também em alguns pacientes que tinham sido tratados com outras drogas antipsicóticas sem resultados satisfatórios. Foi assinalado efeito nítido sôbre o apragmatismo e o embotamento afetivo. Ocorreu ação sedativa nas agitações psicomotoras devendo-se salientar que o resultado positivo se fez quase sempre sentir com dose mínima ( $5-10 \mathrm{mg} /$ dia $)$ já nos primeiros dias de tratamento. Foi ótima a tolerância ao medicamento. Os efeitos colaterais foram mínimos.

Sendo a esquizofrenia em crianças e adolescentes de difícil tratamento, a existência desta nova droga é, na opinião dos autores, uma util contribuição para sua terapêutica.

\section{S U M M A R Y}

Effects of thiothixene $(P-4657$ B) in psychotic children and adolescents.

The effects of thiothixene (P-4657 B) in 34 psychotic children and adolescents were studied; 29 were schizophrenic subjects, 17 had been ill for less than one year and 12 for a longer time. Good results were observed in 20 cases, with evident improvement of the psychiatric symptomatology. 
Tclerance has been very good and side effects minimum. Subsidiary examinations (hemoglobin level and leucocyte count, tests for evaluation of the functional state of the liver and electroencephalogram) were normal.

The thiothixene was observed to have an antipsychotic effect when used as the first treatment, as well as when the patient had already been treated with other antipsychotic drugs without satisfactory result. Remarkable effects were naticed on apragmatism and emotional blunting. Sedative effect was observed on crises of psychomotor instability. Positive result, were almost always noticed with minimum dosage (5-10 $\mathrm{mg}$ per day) in the early stage of treatment.

\section{R E F E R E C I A S}

1. SIMPSON, G. M. \& IQBAL, J. - A preliminary study of thiothixene in chronic schizophrenics. Curr. Therap. Res. 7:697-700, 1965.

2. SIGNORINI, J.; VIEIRA, N.C.; ASSIS, L. M. \& MARTINS, C. - Ensaio clínico com novo psicotrópico (P-4657B) em pacientes hospitalizados. Arq. NeuroPsiquiat. (São Paulo) 25:124-129, 1967.

3. SUGERMAN, A. A.; STOLBERG, J. \& HERRMANN, J. - A pilot study of P-4657B in chronic schizophrenics. Curr. Therap. Res. 7:310-314, 1965.

4. Gallant, D. M.; Bishop, M. P.; TIMMOUS, E. \& GOULD, A. R. Thiothixene (P-4657B): a controlled evaluation in chronic schizophrenics patients. Curr. Therap. Res. 8:153-158, 1966.

5. DELAY, J.; DENIKER, P.; GINESTET, D.; PERIER, M.; PEIONMAONAN, P. \& DALLE, B. - Avaliation clinique précoce d'une nouvelle formule neuroleptique: le thiothixene. An. Méd.-Psychol. 2:192-202, 1 s:66.

6. BAPTISTETE, E.; NAVARRo, E. S.; PENNA, J. M. R.; FONSECA, C. A.; D'AGOSTINHO, E. \& MARTINS, C. - Emprêgo do tiotixene em crianças e adolescentes esquizofrênicos: avaliação clinica. Rev. bras. Psiquiat. 1: $52-59,1967$.

Clínica Psiquiátrica - Faculdade de Medicina da Universidade de São Paulo Caixa Postal 8091 - São Paulo, SP - Brasil 